\title{
IncRNA FGD5 antisense RNA 1 upregulates RORA to suppress hypoxic injury of human cardiomyocyte cells by inhibiting oxidative stress and apoptosis via miR-195
}

\author{
XINYONG CAI $^{1 *}$, PING ZHANG $^{2}$, SHU WANG $^{3 *}$, LANG HONG $^{1 *}$, SONGPING YU $^{1}$, \\ BIN LI $^{1}$, HONG ZENG $^{1}$, XU YANG ${ }^{4}$ and LIANG SHAO ${ }^{1}$ \\ Departments of ${ }^{1}$ Cardiology and ${ }^{2}$ Neurology, Jiangxi Provincial People's Hospital Affiliated to Nanchang University; \\ ${ }^{3}$ Department of Gerontology, The First Affiliated Hospital of Nanchang University, Nanchang, Jiangxi 330006; \\ ${ }^{4}$ Shenzhen Realomics (Biotech), Co., Ltd., Shenzhen, Guangdong 518000, P.R. China
}

Received January 16, 2020; Accepted July 31, 2020

DOI: $10.3892 / \mathrm{mmr} .2020 .11558$

\begin{abstract}
FGD5 antisense RNA 1 (FGD5-AS1) is a long non-coding RNA in acute myocardial infarction (AMI), which is primarily caused by myocardial ischemia-hypoxia. Retinoid acid receptor-related orphan receptor $\alpha$ (RORA) is a key protector in maintaining heart function. However, the roles of FGD5-AS1 and RORA in AMI have not previously been elucidated. The present study investigated the effect and mechanism of FGD5-AS1 and RORA in human cardiomyocyte AC16 cells under hypoxia. Reverse transcription-quantitative PCR and western blotting demonstrated that FGD5-AS1 and RORA were downregulated in the serum of patients with AMI and hypoxia-challenged AC16 cells. Functional experiments were performed via assays, flow cytometry and western blotting. In response to hypoxia, superoxide dismutase (SOD) activity was inhibited, but apoptosis rate and levels of reactive oxygen species and malondialdehyde were promoted in AC16 cells, accompanied by increased Bax and cleaved caspase- 3 expression levels, and decreased SOD2 and glutathione peroxidase 1 expression levels. However, hypoxia-induced oxidative stress and apoptosis in AC16 cells were attenuated by ectopic expression of FGD5-AS1 or RORA. Moreover, silencing RORA counteracted the suppressive role of FGD5-AS1 overexpression in hypoxic injury. FGD5-AS1 controlled RORA expression levels via microRNA-195-5p (miR-195), as confirmed by dual-luciferase reporter and RNA pull-down assays. Consistently, miR-195 knockdown suppressed hypoxia-induced oxidative stress and apoptosis in AC16
\end{abstract}

Correspondence to: Mr. Liang Shao, Department of Cardiology, Jiangxi Provincial People's Hospital Affiliated to Nanchang University, 152 Aiguo Road, Nanchang, Jiangxi 330006, P.R. China E-mail: shaoliang021224@hotmail.com

*Contributed equally

Key words: FGD5 antisense 1, retinoid acid receptor-related orphan receptor $\alpha$, microRNA-195, hypoxia, cardiomyocyte cells, which was abrogated by downregulating FGD5-AS1 or RORA. In conclusion, FGD5-AS1 modulated hypoxic injury in human cardiomyocytes partially via the miR-195/RORA axis, suggesting FGD5-AS1 as a potential target in interfering with the progression of AMI.

\section{Introduction}

Acute myocardial infarction (AMI) is a common cardiovascular disease that is the leading cause of morbidity and mortality among 240 causes of death between 1990 and 2013 globally across all ages and both sexes (1). Myocardial ischemia-hypoxia is the primary cause of AMI (2), and ischemia-hypoxia generates cellular stresses, such as oxidative stress, that lead to irreversible myocardial injuries (3). Mitochondria are prone to cellular stresses, including hypoxia, and mitochondrial dysfunction is associated with the intrinsic pathway of apoptosis in cardiomyocytes under hypoxia $(3,4)$. Thus, lowering oxidative damage may be a promising approach to salvage ischemic myocardium $(5,6)$.

Non-coding (nc)RNAs, comprising microRNAs (miRNA or miR) and long ncRNAs (lncRNAs), are promising novel targets for the treatment of cardiovascular diseases, including AMI (7). Abnormal ncRNA transcriptions have been identified in ischemic-hypoxic heart and cardiomyocytes $(8,9)$. Moreover, ncRNAs are associated with myocardial ischemia-reperfusion (I/R), the primary therapeutic choice for AMI (10). In addition, mutual interaction between IncRNAs, miRNAs and mRNA serves a key role in transcriptional regulation following AMI, and a competing endogenous RNA (ceRNA) network has been identified in patients with AMI (11) as well as I/R-mediated injury (12).

lncRNA FGD5 antisense RNA 1 (FGD5-AS1) is a cancer-associated gene (13-15) and is a key lncRNA in patients with AMI, due to its roles in regulating $>50$ differentially expressed genes, according to the GSE48060 dataset (11). miR-195-5p is a notable oncogene in cancer (16), and has been identified as a cardio- and stress-responsive miRNA that participates in cardiac remodeling $(17,18)$. Retinoid acid receptor-related orphan receptor $\alpha$ (RORA) is an important 
circadian rhythm gene (19) and defends against cardiomyocyte injury in mouse heart cells (20).

The present study aimed to analyze the expression levels of FGD5-AS1 and RORA in patients with AMI using the GSE48060 dataset, and to confirm their roles in a hypoxia-induced model of AMI in human cardiomyocyte AC16 cells. The present study also aimed to identify the association between FGD5-AS1, miR-195 and RORA in regulating oxidative damage of hypoxic AC16 cells.

\section{Materials and methods}

In silico data analysis. The GSE48060 (ncbi.nlm.nih. gov/GSE48060) dataset was downloaded (11). This cohort of blood samples was isolated from 31 patients with AMI and 21 normal controls. The expression levels of FGD5-AS1 and RORA mRNA were compared between AMI and normal cases and results were plotted as a scatter diagram.

Cell model of AMI. Human cardiomyocyte cell line AC16 (SCC109) was purchased from EMD Millipore, and cultured in DMEM with $10 \%$ FBS (both HyClone; Cytiva) at $37^{\circ} \mathrm{C}$ in normoxic conditions $\left(21 \% \mathrm{O}_{2}, 5 \% \mathrm{CO}_{2}\right.$ and $\left.74 \% \mathrm{~N}_{2}\right)$. For hypoxia treatment, AC16 cells were starved in FBS-free DMEM for $12 \mathrm{~h}$, and then incubated in hypoxic conditions $\left(1 \% \mathrm{O}_{2}, 5 \% \mathrm{CO}_{2}\right.$ and $\left.94 \% \mathrm{~N}_{2}\right)$ for $12 \mathrm{~h}$.

Cell transfection. For overexpression, the full length of FGD5-AS1 (ENSG00000225733) and coding domain sequence of RORA (NM_134261) were separately inserted into pEGFP-C1 (Clontech Laboratories, Inc.), generating overexpression vectors oe-FGD5-AS1 and oe-RORA, respectively; miR-195 (5'-UAGCAGCACAGAAAUAUUGGC-3') and miR-NC mimics (5'-GUCCAGUGAAUUCCCAG-3') were provided by Sangon Biotech Co., Ltd. For silencing, siRNA against RORA (isoform a) (si-RORA; 5'-TACGTGTGAAGG CTGCAAGGGC-3') and FGD5-AS1 (si-FGD5-AS1; 5'-UUG GUCGUUGUCAACUUCCCA-3'), and anti-RNA against miR-195 (anti-miR-195; 5'-GCCAAUAUUUCUGUGCUG CUA-3') were purchased from Sangon Biotech Co., Ltd., as well as the negative controls (NC) si-NC (5'-UUCUCCGAA CGUGUCACGUTT-3') and anti-NC (5'-UUCUCCGAACGU GUCACGUTT-3'). The transfection was performed in AC16 cells in 6-well plates using Lipofectamine ${ }^{\circledR} 3000$ reagent (Invitrogen; Thermo Fisher Scientific, Inc.), according to the manufacturer's instructions. Briefly, $2 \mu \mathrm{g}$ overexpression vectors and $30 \mathrm{nM}$ oligonucleotides diluted in Opti-MEM ${ }^{\mathrm{TM}}$ Medium (Invitrogen; Thermo Fisher Scientific, Inc.) were mixed with 3.5 $\mu$ l Lipofectamine 3,000 reagent diluted in Opti-MEM Medium. The transfection efficiency was assessed prior to hypoxia treatment, following transfection for $36 \mathrm{~h}$.

Reverse transcription-quantitative $(R T-q) P C R$. Total RNA in AC16 cells was extracted using RNAiso Plus (Takara Bio, Inc.) prior to complementary DNA synthesis using PrimeScript ${ }^{\mathrm{TM}}$ RT MasterMix kit (Invitrogen; Thermo Fisher Scientific, Inc.). Reverse transcription was performed at $25^{\circ} \mathrm{C}$ for $10 \mathrm{~min}, 42^{\circ} \mathrm{C}$ for $15 \mathrm{~min}$ and $85^{\circ} \mathrm{C}$ for $5 \mathrm{~min}$. The RNA expression levels were analyzed using SYBR-Green (Takara Bio, Inc.) on a 7900HT Fast Real-Time PCR system (Thermo Fisher Scientific, Inc.) and the following primers: FGD5-AS1 forward, 5'-AGAAGC GGAGGGGTGAAAAT-3' and reverse, 5'-CCGCCTTATAGT TGGCCCTC-3'; miR-195 forward, 5'-GTCTAGCAGCAC AGAAATA-3' and reverse, 5'-GTGCAGGGTCCGAGGT-3'; RORA forward, 5'-AAAAACATGGAGTCAGCTCCG-3' and reverse, 5'-AGTGTTGGCAGCGGTTTCTA-3'; GAPDH forward, 5'-CAGTCACTACTCAGCTGCCA-3' and reverse, 5'-GAGGGTGCTCCGGTAG-3'; U6 forward, 5'-CTCGCT TCGGCAGCACA-3' and reverse, 5'-AACGCTTCACGAA TTTGCGT-3'; 18S ribosomal N1 RNA (18S rRNA) forward, 5'-CTGGATACCGCAGCTAGGAA-3' and reverse, 5'-GAA TTTCACCTCTAGCGGCG-3'. The qPCR thermocycling conditions were as follows: $95^{\circ} \mathrm{C}$ for $15 \mathrm{~min}, 40$ cycles at $94^{\circ} \mathrm{C}$ for $15 \mathrm{sec}, 60^{\circ} \mathrm{C}$ for $30 \mathrm{sec}$ and $72^{\circ} \mathrm{C}$ for $60 \mathrm{sec}$. The results were calculated via the $2^{-\triangle \Delta C q}$ method (21), and normalized to the expression levels of GAPDH (for FGD5-AS1 and RORA) or U6 (for miR-195). PCR reactions were repeated four times. For subcellular distribution of FGD5-AS1, Nuclear/Cytosol Fraction Kit (BioVision, Inc.) was utilized to obtain RNA in cytoplasm and nuclear fractions, followed by normal RT-qPCR analysis as aforementioned.

Western blotting. AC16 cells were lysed in radioimmunoprecipitation assay buffer (Thermo Fisher Scientific, Inc.), and the supernatant containing total protein samples was collected following centrifugation at $12,000 \mathrm{x}$ g at $4{ }^{\circ} \mathrm{C}$ for $15 \mathrm{~min}$. Following separation of $20 \mu \mathrm{g}$ protein samples (determined by Bradford method) via SDS-PAGE on 12-15\% gels, the proteins were electrophoretically transferred onto PVDF membranes (EMD Millipore). Following blocking with $3 \%$ bovine serum albumin at room temperature for $1 \mathrm{~h}$, the proteins were incubated with the following primary antibodies at $4^{\circ} \mathrm{C}$ overnight: RORA (1:500; cat. no. ab60134), superoxide dismutase 2 (SOD2; 1:10,000; cat. no. ab118340), glutathione peroxidase1 (GPx1; 1:500; cat. no. ab50427), Bax (1:1,000; cat. no. ab53154), cleaved caspase-3 (cleaved casp-3; 1:500; cat. no. ab49822) and GAPDH (1:2,500; cat. no. ab9485). The HRP-conjugated secondary antibodies [goat anti-rabbit (1:50,000; cat. no. ab205718) and rabbit anti-goat (1:20,000; cat. no. ab6741)] were used to make protein bands immunoreactive at room temperature for $1 \mathrm{~h}$ for further visualization using via chemiluminescence (EMD Millipore). The gray intensity was quantified using ImageJ version 1.8.0 software (National Institutes of Health). The relative protein expression levels were normalized to those of GAPDH. All antibodies were obtained from Abcam.

Flow cytometry (FCM). Hypoxia-treated AC16 cells were analyzed using Annexin V-FITC early apoptosis detection kit (Cell Signaling Technology, Inc.) according to the manufacturer's instructions. The stained cells were analyzed on a BD Accuri ${ }^{\mathrm{TM}}$ C6 Flow Cytometer (BD Biosciences), and apoptotic cells (\%) were the percentage of cells in Annexin $\mathrm{V}^{+} / \mathrm{PI}^{+}$ and Annexin $\mathrm{V}^{+} / \mathrm{PI}^{-}$quadrants analyzed on CellQuest ${ }^{\mathrm{TM}}$ Pro software 5.1 (BD Biosciences).

Reactive oxygen species (ROS), malonaldehyde (MDA) and SOD assays. Generation of ROS was determined via the 2',7'-dichlorofluorescin diacetate (DCFH-DA) method. In brief, hypoxia-treated AC16 cells were exposed to $10 \mu \mathrm{M}$ 

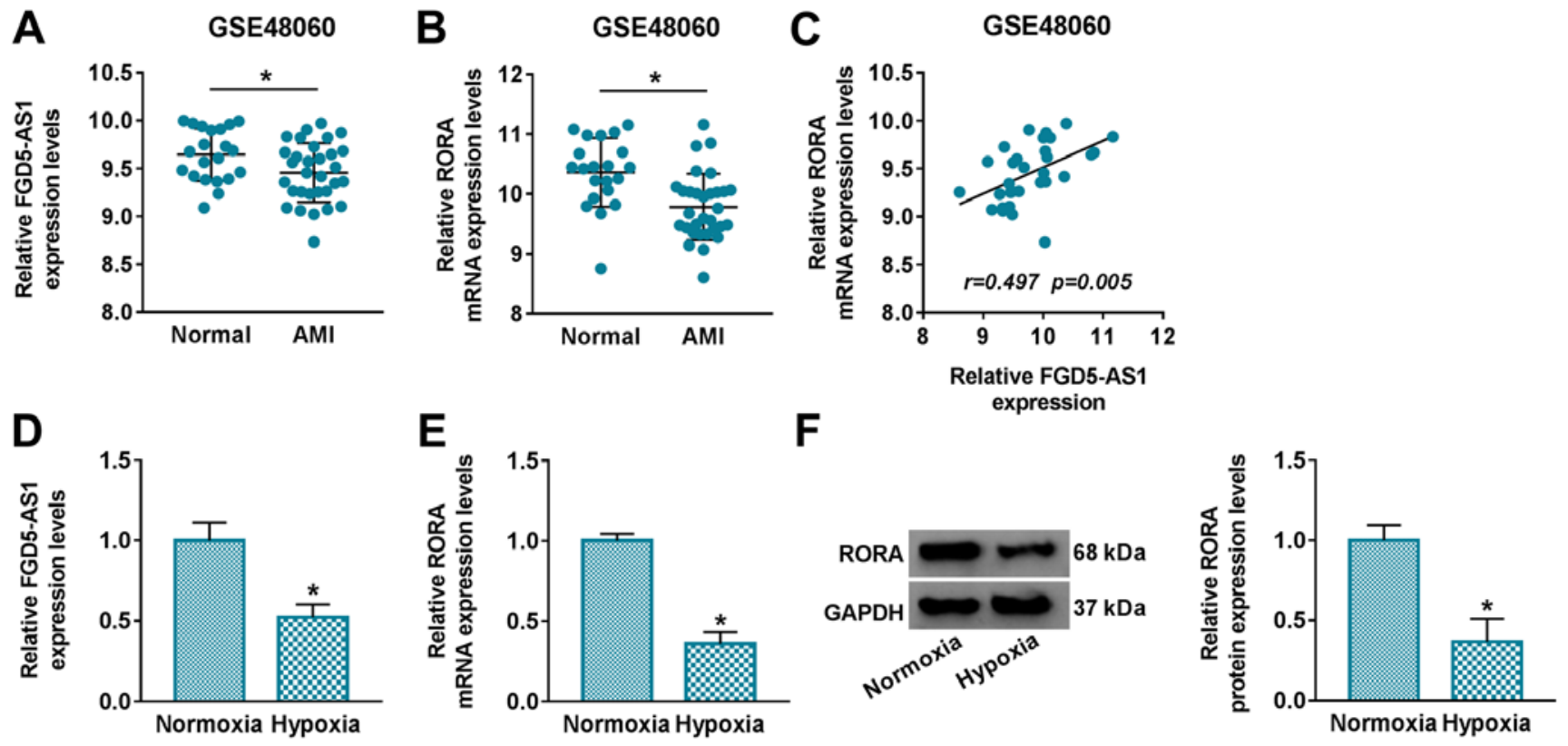

Figure 1. Expression levels of 1ncRNA FGD5-AS1 and RORA in patients with AMI and hypoxia-treated cardiomyocytes. Relative expression levels of (A) FGD5-AS1 and (B) RORA mRNA in the serum of patients with AMI ( $\mathrm{n}=31)$ and normal controls $(\mathrm{n}=21)$ were analyzed using the GSE48060 dataset. (C) Pearson rank correlation analysis confirmed the correlation between FGD5-AS1 and RORA expression levels. Reverse transcription-quantitative PCR detected expression levels of (D) FGD5-AS1 and (E) RORA mRNA in human cardiomyocyte AC16 cells treated with hypoxia or normoxia for $12 \mathrm{~h}$. (F) Western blotting measured RORA protein expression levels in hypoxic and normoxic cells. ImageJ software determined the relative RORA protein expression levels by analyzing gray density. ${ }^{*} \mathrm{P}<0.05$ vs. control group or as indicated. lncRNA, long non-coding RNA; FGD5-AS1, FGD5 antisense 1; RORA, retinoid acid receptor-related orphan receptor $\alpha$; AMI, acute myocardial infarction.

DCFH-DA (ROS probe) for $1 \mathrm{~h}$ at $37^{\circ} \mathrm{C}$ in the dark. Then, the stained cells were analyzed using a Tecan Infinite M200 fluorescence plate reader (Tecan Group Ltd.), and fluorescence intensity was measured at $525 \mathrm{~nm}$ with an excitation wavelength of $488 \mathrm{~nm}$. MDA and SOD levels were measured using MDA Assay (Colorimetric/Fluorometric) (cat. no. ab118970; Abcam) and human SOD2 ELISA kits (cat. no. ab178012; Abcam) according to the manufacturer's instructions. The absorbance was read at 532 (for MDA) or $450 \mathrm{~nm}$ (for SOD).

Dual-luciferase reporter assay. StarBase software version 2.0 (http://starbase.sysu.edu.cn/index.php) was used to predict the potential binding site of miRNAs on both FGD5-AS1 (starbase.sysu.edu.cn/lncRNA) and RORA (starbase.sysu.edu. $\mathrm{cn} / \mathrm{mRNA}$ ). A total of two paired recombinant reporter vectors psiCHECK-2 (Promega Corporation) were constructed, carrying wild-type (WT) or mutant (MUT) FGD5-AS1 fragment and the 3'-untranslated region (3'UTR) of RORA. AC16 cells in 24-well plates were co-transfected with vectors and miR-195 or miR-NC mimic using Lipofectamine 3000 reagent (Invitrogen; Thermo Fisher Scientific, Inc.) according to the manufacturer's instructions. Following transfection for $48 \mathrm{~h}$, the luciferase activity of psiCHECK-2 vector was measured via Dual-Glo ${ }^{\circledR}$ dual-luciferase kit (cat. no. E2920; Promega Corporation). The ratio of firefly luciferase activity compared to that of Renilla was calculated.

RNA pull-down assay. miRNA mimics were labelled with bio-16-UTP using T7 High Yield Transcription kit (Thermo Fisher Scientific, Inc.). The bio-16-UTP-labelled miR-195 (bio-miR-195) or miR-NC mimics (bio-miR-NC) were conjugated with streptavidin magnetic beads at $4^{\circ} \mathrm{C}$ for $2 \mathrm{~h}$. After centrifugation at 2,000 $\mathrm{x}$ g for $1 \mathrm{~min}$ at $4^{\circ} \mathrm{C}$, the RNA-beads were collected and lysed in RNAiso Plus (Takara Bio, Inc.) supplemented with $50 \mu \mathrm{g} / \mathrm{ml}$ Proteinase $\mathrm{K}$ (Thermo Fisher Scientific, Inc.) at $4^{\circ} \mathrm{C}$ for $2 \mathrm{~h}$. The supernatant was collected for total RNA samples after centrifugation for $1 \mathrm{~min}$ at $2,000 \mathrm{x} \mathrm{g}$ at $4^{\circ} \mathrm{C}$, and gene abundance was measured using RT-qPCR as aforementioned.

Statistical analysis. Data are presented as the mean \pm standard deviation from at least three independent biological repeats. The statistical analysis was performed using GraphPad Prism 6.0 software (GraphPad Software, Inc.), and the P-value was calculated using unpaired Student's t-test (two-tailed) or one-way ANOVA. Tukey's post hoc test was performed following ANOVA. Pearson rank correlation analysis was used to evaluate the correlation between gene expression levels. $\mathrm{P}<0.05$ was considered to indicate a statistically significant difference.

\section{Results}

FGD5-AS1 and RORA are downregulated in patients with AMI and hypoxia-induced human cardiomyocytes. Analysis of the GSE48060 dataset demonstrated that FGD5-AS1 and RORA were significantly downregulated in the serum of patients with AMI compared with healthy normal controls (Fig. 1A and B). Moreover, Pearson rank correlation analysis confirmed a positive linear correlation between FGD5-AS1 and RORA expression levels in patients with AMI $(r=0.497$; $\mathrm{P}=0.005$ ) (Fig. 1C). These data indicated a potential role of FGD5-AS1 and RORA in myocardial damage. In order to 


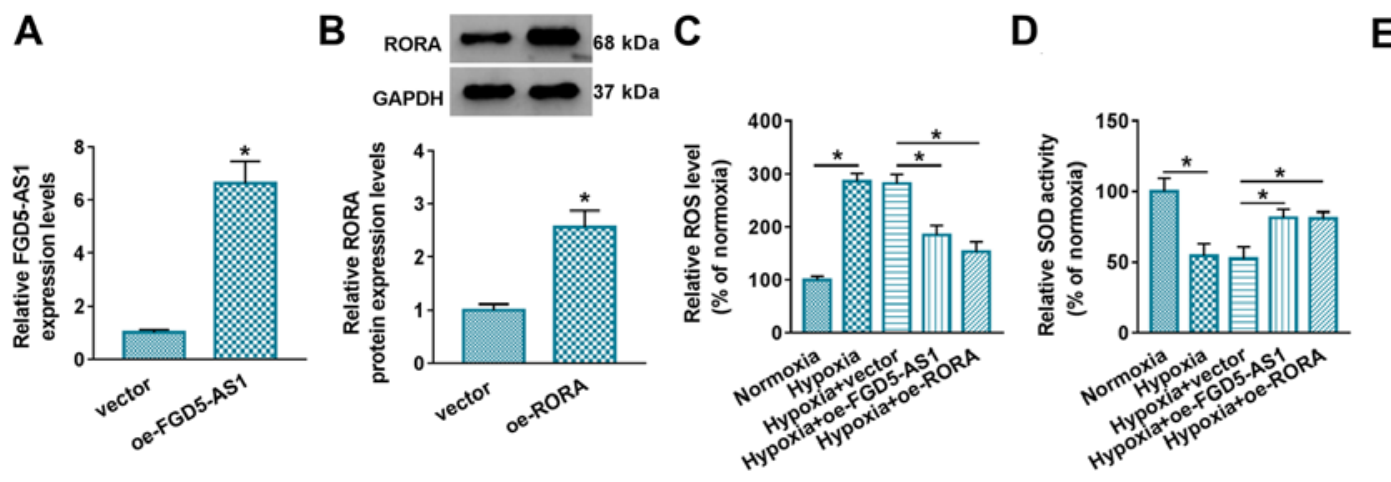

E
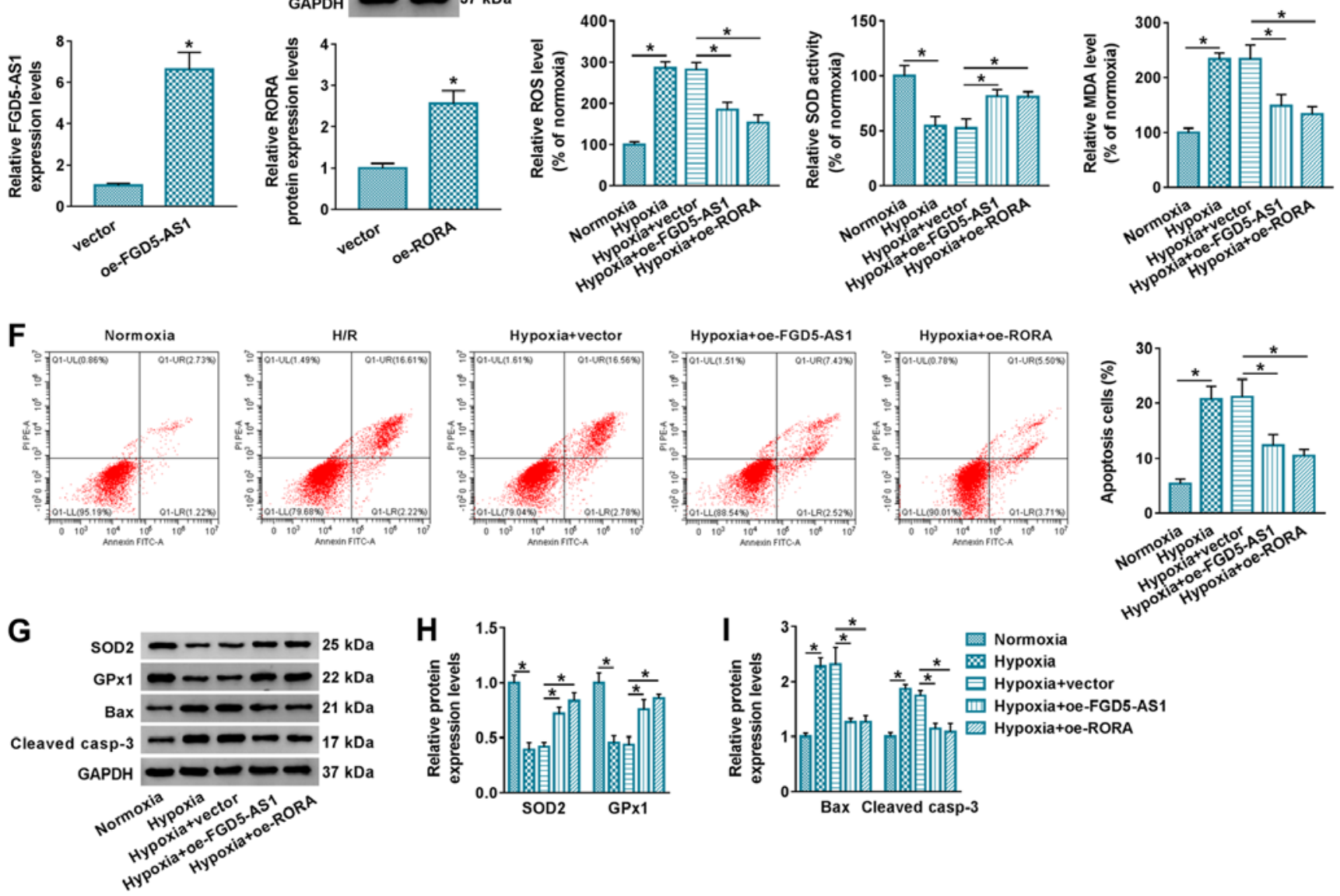

Figure 2. Effect of FGD5-AS1 and RORA on hypoxic injury in cardiomyocytes. (A) Reverse transcription-quantitative PCR determined FGD5-AS1 levels in AC16 cells transfected with oe-FGD5-AS1 or empty vector. (B) Western blotting determined RORA protein level in AC16 cells transfected with oe-RORA or empty vector. AC16 cells were transfected with oe-FGD5-AS1, oe-RORA or vector, and then treated with hypoxia for $12 \mathrm{~h}$. Commercial kits examined the relative levels of (C) ROS, (D) SOD and (E) MDA. (F) Flow cytometry evaluated apoptotic cells in Annexin V-FITC $/ \mathrm{PI}^{+}$and Annexin V-FITC $/$PI $^{-}$quadrants. (G) Western blotting assessed the protein expression levels of SOD2, GPx1, Bax and cleaved casp-3. ImageJ software determined the relative protein expression levels of (H) SOD2, GPx1 and (I) Bax and cleaved casp-3 by analyzing gray density. ${ }^{~} \mathrm{P}<0.05$ vs. control group or as indicated. FGD5-AS1, FGD5 antisense 1; RORA, retinoid acid receptor-related orphan receptor $\alpha$; oe, overexpression; ROS, reactive oxygen species; SOD, superoxide dismutase; MDA, malondialdehyde; GPx1, glutathione peroxidase 1; cleaved casp-3, cleaved caspase-3.

investigate their role in myocardial damage, the expression levels of FGD5-AS1 and RORA in a cell model of AMI were further evaluated. Relative expression levels of FGD5-AS1 and RORA were revealed to be significantly decreased in hypoxia-challenged AC16 cells (Fig. 1D and F).

Ectopic expression levels of FGD5-AS1 or RORA suppress hypoxia-induced oxidative stress and apoptosis in cardiomyocytes. Next, the functional role of FGD5-AS1 and RORA overexpression in an AMI cell model was investigated. The overexpression of FGD5-AS1 and RORA was induced in AC16 cells via transfection (Fig. 2A and B). In response to hypoxic stress, ROS and MDA levels were highly induced (Fig. 2C and E), but SOD activity and the expression levels of SOD2 and GPx1 were significantly inhibited in AC16 cells (Fig. 2D, G and H), along with promotion of apoptosis and the expression levels of Bax and cleaved casp-3 (Fig. 2F, G and I). These results indicated that hypoxia induced oxidative stress and apoptosis of AC16 cells. However, transfection of oe-FGD5-AS1 and oe-RORA separately attenuated hypoxia-induced oxidative stress and apoptosis (Fig. 2C-I).
These results indicated a suppressive role of FGD5-AS1 and RORA in hypoxic injury in AC16 cells.

Silencing RORA abrogates the suppressive role of FGD5-ASI overexpression in hypoxic injury in cardiomyocytes. It was next determined whether there was an interaction between FGD5-AS1 and RORA. FGD5-AS1 ectopic expression levels resulted in RORA upregulation in AC16 cells (Fig. 3A). Therefore, the effect of RORA knockdown on FGD5-AS1 overexpression was evaluated. RORA knockdown was performed via siRNA transfection (Fig. 3B). Assays demonstrated that the lower levels of ROS and MDA in hypoxic AC16 cells transfected with oe-FGD5-AS1 were restored, and higher SOD activity was diminished following RORA silencing (Fig. 3C-E), accompanied by decreased SOD2 and GPx1 expression levels (Fig. 3G and H). FCM demonstrated that FGD5-AS1-mediated apoptosis inhibition in hypoxic AC16 cells was relieved by blocking RORA (Fig. 3F), along with increased Bax and cleaved casp-3 expression levels (Fig. 3G and I). These results indicated that RORA knockdown partially abolished the suppressive effect of 
A

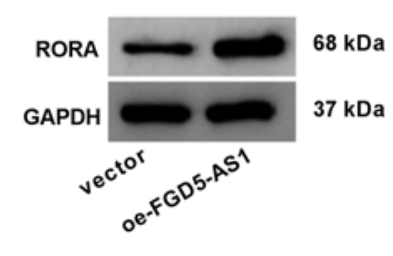

C

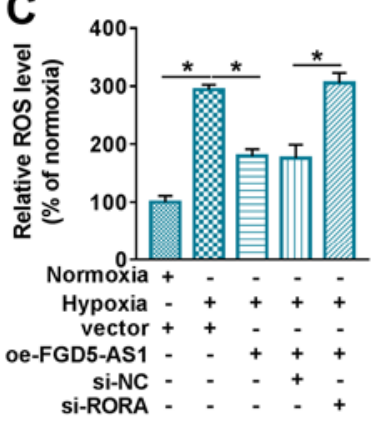

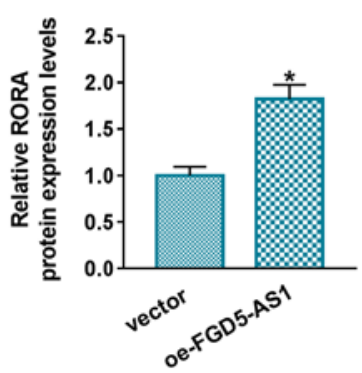

D

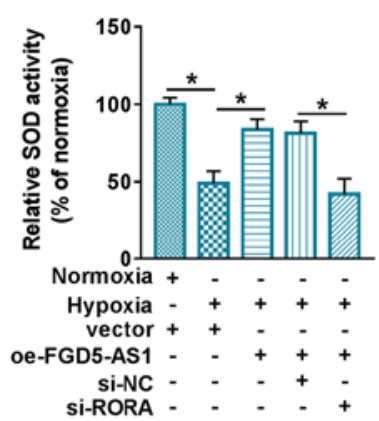

B

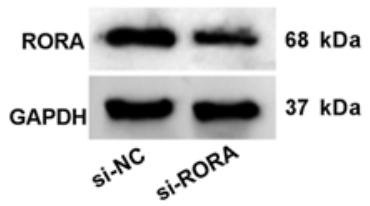

E

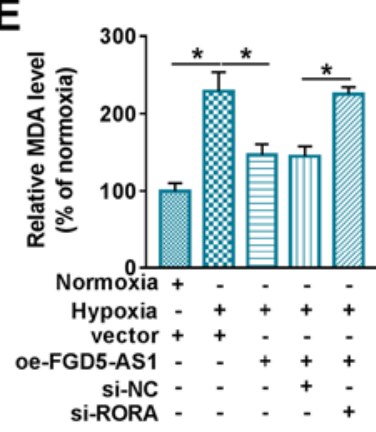

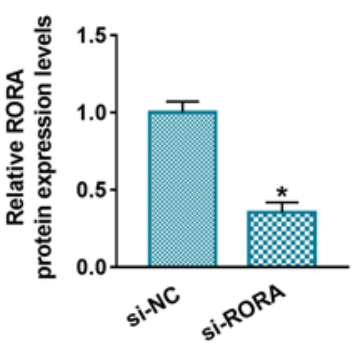

F

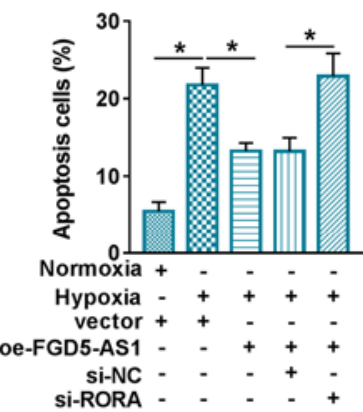

G

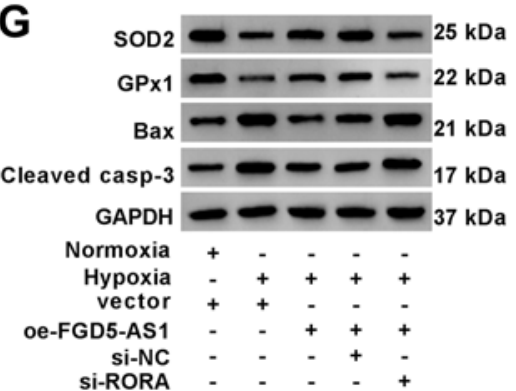

H

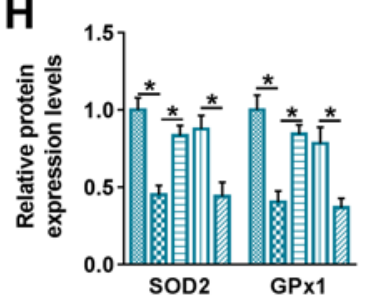

I

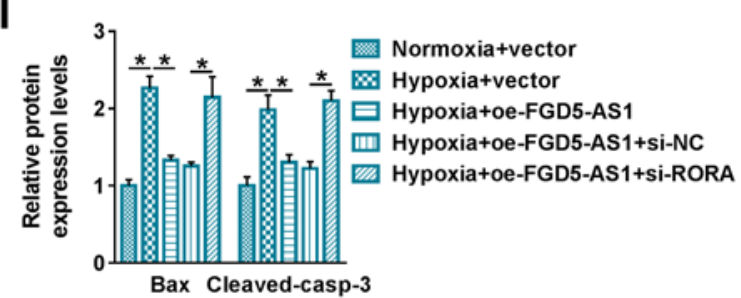

Figure 3. Interfering RORA abrogates the role of FGD5-AS1 overexpression in hypoxia-induced cardiomyocyte injury. Western blotting measured RORA protein expression levels in AC16 cells transfected with (A) oe-FGD5-AS1 or vector and (B) si-RORA or si-NC. Following hypoxia treatment for $12 \mathrm{~h}$, assays were performed to assess the relative levels of (C) ROS, (D) SOD and (E) MDA. (F) Flow cytometry was performed to evaluate apoptotic cells. (G) Western blotting was performed, and then semi-quantified to assess the relative protein expression levels of (H) SOD2, GPx1, (I) Bax, and cleaved casp-3. "P<0.05 vs. control group or as indicated. RORA, retinoid acid receptor-related orphan receptor $\alpha$; FGD5-AS1, FGD5 antisense 1; oe, overexpression; siRNA, small interfering RNA; NC, negative control; ROS, reactive oxygen species; SOD, superoxide dismutase; MDA, malondialdehyde; GPx1, glutathione peroxidase 1; cleaved casp-3, cleaved caspase-3.

FGD5-AS1 on oxidative stress and apoptosis in hypoxic AC16 cells.

miR-195 is sponged by FGD5-AS1, and RORA is a target of $m i R-195$. Analysis of the relative expression levels of FGD5-AS1 in subcellular fractions demonstrated that FGD5-AS1 was predominantly present in the cytoplasm rather than the nucleus, which was similar to the distribution of 18S rRNA (Fig. 4A). Thus, a potential ceRNA pathway between FGD5-AS1, RORA and miRNAs was hypothesized. StarBase software was utilized to predict the potential binding site of miRNAs on both FGD5-AS1 and RORA. miR-195, a cardio-miRNA and stress-responsive miRNA that plays a role in regulating cardiac remodeling $(17,18)$, was suggested to be complementary to both FGD5-AS1 and RORA, thus this miRNA was selected for further investigation (Fig. 4B). The miR-195 or miR-NC mimic was exogenously administrated in AC16 cells (Fig. 4C), and dual-luciferase reporter assays were performed. Relative luciferase activity of FGD5-AS1 WT was significantly attenuated by miR-195 mimic transfection compared with miR-NC mimic transfection, whereas FGD5-AS1 MUT luciferase activity exhibited little difference in the presence of miR-195 or miR-NC mimic (Fig. 4D and E). FGD5-AS1 and RORA expression levels were enriched in the bio-miR-195-mediated RNA complex in AC16 cells (Fig. 4F). oe-FGD5-AS1 transfection resulted in FGD5-AS1 overexpression and miR-195 downregulation (Figs. 2A and 4H); si-FGD5-AS1 transfection decreased FGD5-AS1 and upregulated miR-195 (Fig. 4G and H). In addition, miR-195 overexpression via mimic transfection downregulated RORA protein levels in AC16 cells, and this effect was further modulated by FGD5-AS1 (Fig. 4I). These results demonstrated that FGD5-AS1 sponged miR-195 to modulate RORA in AC16 cells. miR-195 was highly induced in response to hypoxic stress (Fig. 4J).

Upregulating FGD5-AS1 or RORA abolishes the diminishing effect of miR-195 knockdown on hypoxia-induced oxidative stress and apoptosis in cardiomyocytes. The role of miR-195 knockdown was further assessed in hypoxic AC16 cells via anti-RNA transfection. Silencing efficiency of anti-miR-195 was determined via RT-qPCR (Fig. 5A). Commercial assays demonstrated that the promotion of ROS and MDA levels was reversed following anti-miR-195 transfection in hypoxic 


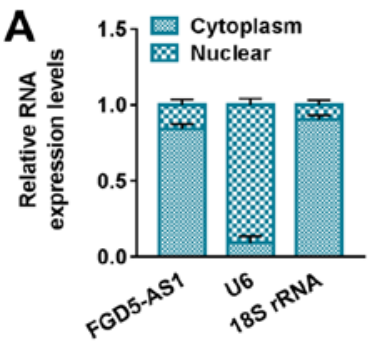

B

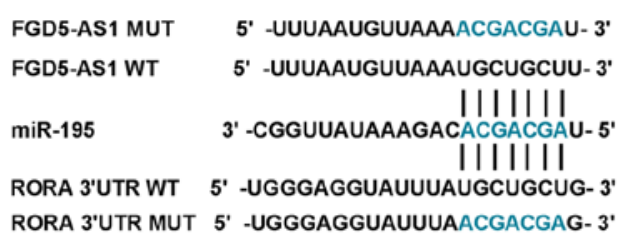

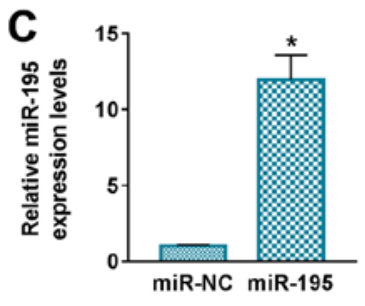
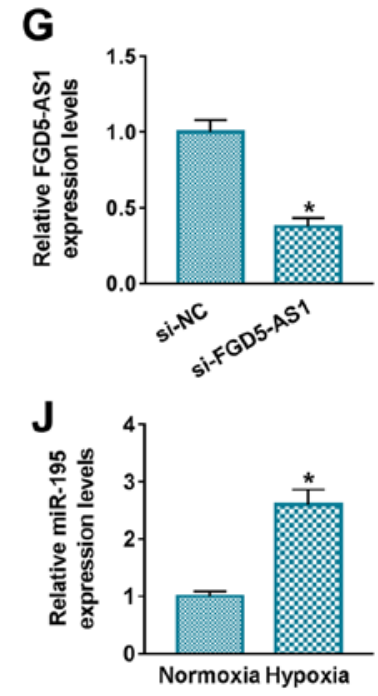
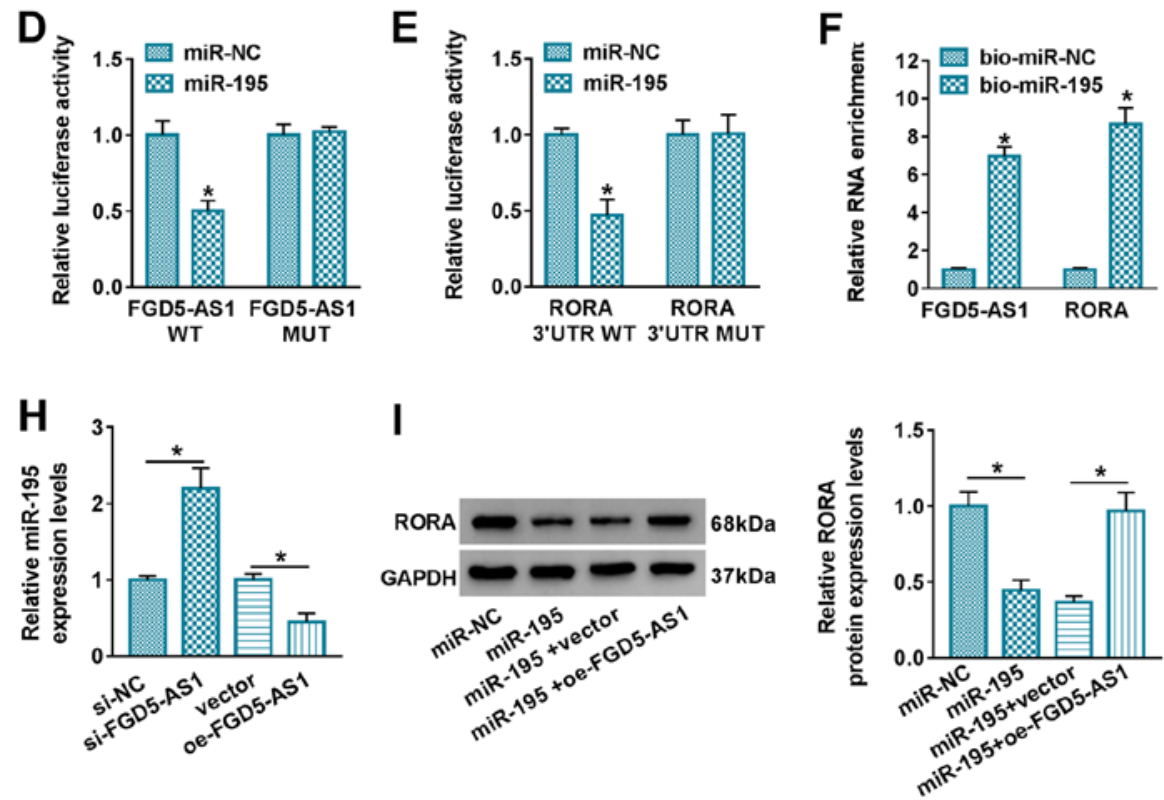

Figure 4. Interaction between FGD5-AS1, RORA and miR-195 in cardiomyocytes. (A) RT-qPCR assessed the relative RNA expression levels in the cytoplasm and nuclear fraction of AC16 cells. (B) StarBase software predicted the putative binding sites of miR-195 on WT and MUT FGD5-AS1 and RORA 3'UTR. (C) RT-qPCR determined the relative miR-195 expression levels in AC16 cells transfected with miR-195 or miR-NC mimic. Dual-luciferase reporter assay measured the relative luciferase activity of AC16 cells co-transfected with miR-195/NC and vectors carrying (D) FGD5-AS1 WT/MUT or (E) RORA 3'UTR WT/MUT. (F) RNA pull-down and qPCR assays determined the relative enrichment of FGD5-AS1 and RORA in AC16 cells transfected with bio-miR-195 or bio-miR-NC. RT-qPCR determined (G) the relative FGD5-AS1 expression levels in AC16 cells transfected with si-FGD5-AS1 or si-NC and (H) the relative miR-195 expression levels in AC16 cells transfected with si-FGD5-AS1, si-NC, oe-FGD5-AS1 or vector. (I) Western blotting assessed the relative RORA protein expression levels in AC16 cells transfected with miR-195 or miR-NC, and co-transfected with miR-195 and oe-FGD5-AS1 or vector. (J) RT-qPCR detected the relative miR-195 expression levels in hypoxic and normoxic cells. " $\mathrm{P}<0.05$ vs. control group or as indicated. FGD5-AS1, FGD5 antisense 1; RORA, retinoid acid receptor-related orphan receptor $\alpha$; miR, microRNA; RT-qPCR, reverse transcription-quantitative PCR; WT, wild-type; MUT, mutant; UTR, untranslated region; NC, negative control; bio, biotin-labeled; siRNA, small interfering RNA; oe, overexpression; 18S rRNA, 18S ribosome RNA.

AC16 cells, as well as decreased SOD activity (Fig. 5B-D), and increased SOD2 and GPx1 expression levels (Fig. 5F and G). FCM demonstrated that hypoxia-mediated apoptosis of AC16 cells was suppressed by miR-195 knockdown, as evidenced by decreased apoptosis rate (Fig. 5E) and the expression levels of Bax and cleaved casp-3 (Fig. 5F and H). These results revealed that miR-195 knockdown alleviated hypoxic injury in AC16 cells. Notably, the presence of either si-FGD5-AS1 or si-RORA counteracted the biological role of miR-195 knockdown in hypoxic AC16 cells (Fig. 5B-H). These results indicated a novel FGD5-AS1/miR-195/RORA ceRNA pathway underlying hypoxic injury in cardiomyocytes in vitro.

\section{Discussion}

Oxidative stress occurs in cardiomyocyte injury, such as acute ischemia-hypoxia and reperfusion/reoxygenation-mediated injury (22). Previous research has demonstrated that lncRNAs are abnormally expressed in ischemia-hypoxia injury and can affect proliferation, autophagy, migration, invasion and apoptosis of cardiomyocytes $(23,24)$, as well as the inflammatory response (25). In terms of oxidative stress, Su et al (26) reported that lncRNA taurine upregulated 1 knockdown improved viability and inhibited apoptosis and ROS production in $\mathrm{H}_{2} \mathrm{O}_{2}$-induced hypoxic cardiomyocytes via targeting the miRNA-132-3p/histone deacetylase 3 axis. Jiao et al (27) demonstrated that lncRNA ZNFX antisense RNA 1 induced mitochondrial apoptosis via cytosolic $\mathrm{Ca}^{2+}$ overload in AMI mice. However, little is currently known about the association between lncRNAs and oxidative damage in ischemic/hypoxic cardiomyocytes; further investigation is therefore required. The present study confirmed a protective effect of FGD5-AS1 against oxidative stress and apoptosis in a hypoxia-induced model of AMI in AC16 cells via targeting the miR-195/RORA axis.

Downregulation of FGD5-AS1 was observed in the serum of patients with AMI; this finding was consistent with a previous study (11) in which FGD5-AS1, along with other lncRNAs, was identified as key regulator in AMI by regulating $>50$ differentially expressed genes, and an lncRNA-mRNA co-expression network in AMI was identified. The present study confirmed 
A

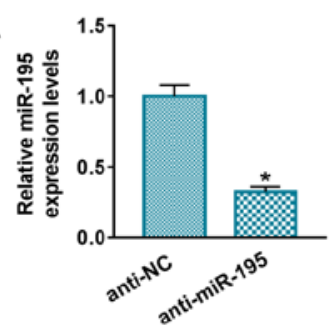

E

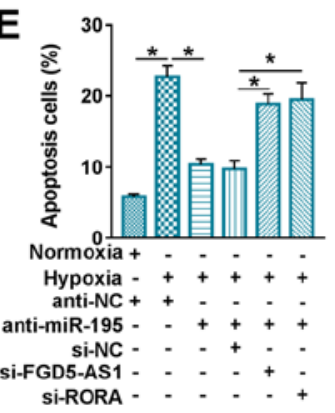

B

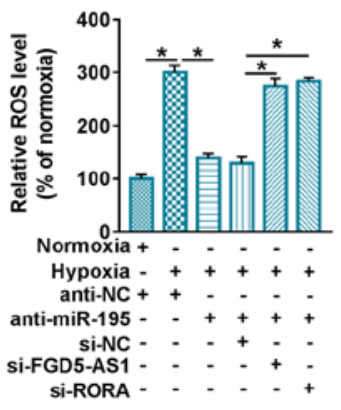

$\mathbf{F}$

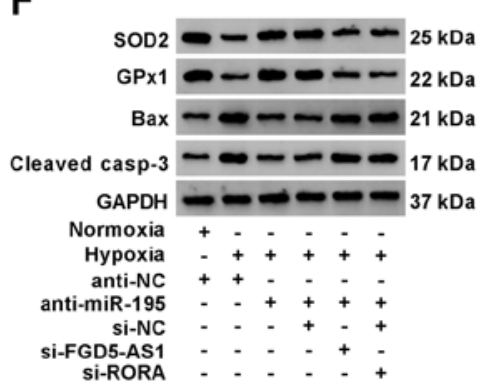

C

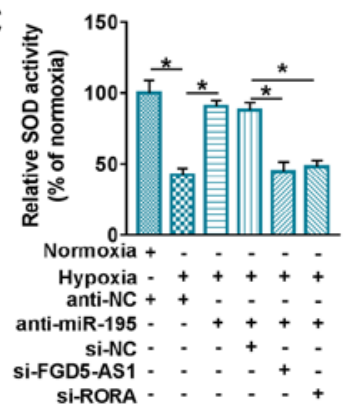

G

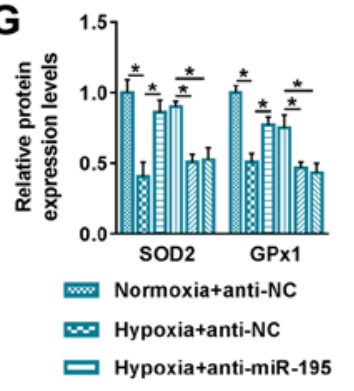

D

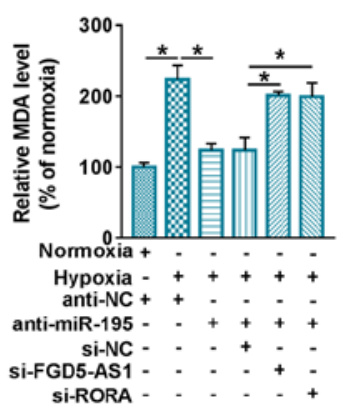

H

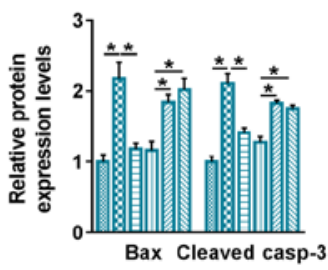

Hypoxia+anti-miR-195+si-NC

Hypoxia+anti-miR-195+si-FGD5-AS1

Hypoxia+anti-miR-195+si-RORA

Figure 5. Interfering FGD5-AS1 or RORA abolishes the role of miR-195 knockdown in hypoxia-induced cardiomyocyte injury. (A) Reverse transcriptionquantitative PCR detected the relative miR-195 expression levels in AC16 cells transfected with anti-miR-195 or anti-NC. AC16 cells were transfected with anti-miR-195 or anti-NC, or co-transfected with anti-miR-195 and si-FGD5-AS1, si-RORA or si-NC, followed by hypoxia treatment for 12 h. Assays were performed to assess the relative levels of (B) ROS, (C) SOD and (D) MDA. (E) Flow cytometry evaluated apoptotic cells. (F) Western blotting was performed, and then semi-quantified to assess the relative protein expression levels of (G) SOD2, GPx1, (H) Bax and cleaved casp-3. "P<0.05 vs. control group or as indicated. FGD5-AS1, FGD5 antisense 1; RORA, retinoid acid receptor-related orphan receptor $\alpha$; miR, microRNA; NC, negative control; siRNA, small interfering RNA; ROS, reactive oxygen species; SOD, superoxide dismutase; MDA, malondialdehyde; GPx1, glutathione peroxidase 1; cleaved casp-3, cleaved caspase-3.

that FGD5-AS1 was downregulated in hypoxia-challenged AC16 cells, and that overexpression of FGD5-AS1 attenuated ROS and MDA levels, the apoptosis rate and expression levels of Bax and cleaved casp-3, but increased SOD activity and the expression levels of SOD2 and GPx1 in hypoxic AC16 cells. These outcomes indicated a suppressive effect of FGD5-AS1 in hypoxia-induced oxidative injury of cardiomyocytes. Chen et al (28) reported a protective role of FGD5-AS1 in lipopolysaccharide-induced inflammatory injury in human periodontal ligament cells, as evidenced by counteraction of cell apoptosis and pro-inflammatory cytokine secretion via the $\mathrm{NF}-\kappa \mathrm{B}$ pathway. The role of FGD5-AS1 in inflammation in AMI requires further investigation, although ischemia-hypoxia is known to highly induce an inflammatory response in AMI (29).

FGD5-AS1 has been revealed to sponge a number of miRNAs, such as miRNA-142-3p, miRNA-125a-3p, miRNA-302e $(13,30)$ and miR-195, to modulate their cellular concentrations. miR-195 was revealed to be involved in the cell cycle, apoptosis and proliferation in multiple diseases, including heart failure (31). In heart cells, miR-195 may be a promising therapeutic strategy for myocardial injury, such as hypoxia- and $\mathrm{H}_{2} \mathrm{O}_{2}$-evoked myocardial ischemia $(32,33)$, I/R injury (34), angiotensin II- and isoprenaline-induced cardiomyocyte hypertrophy $(35,36)$, streptozotocin-mimicked diabetic cardiomyopathy (37) and palmitate-activated lipotoxic cardiomyopathy (38). The present study detected high expression levels of miR-195 in hypoxic AC16 cells, which was consistent with the results of a previous study (32). Silencing of miR-195 may attenuate hypoxia-induced oxidative stress and apoptosis of AC16 cells. Zhang et al (33) reported that miR-195 overexpression suppressed cell viability, and promoted cell apoptosis in HCMs under $\mathrm{H}_{2} \mathrm{O}_{2}$ stimulation; Hang et al (32) demonstrated that miR-195 deletion improved cell viability and decreased apoptosis of primary cardiomyocytes following hypoxia or $\mathrm{H}_{2} \mathrm{O}_{2}$ treatment. Furthermore, it was discovered in the present study that miR-195 expression levels were highly induced by hypoxic stress, which is consistent with the effects of $\mathrm{H}_{2} \mathrm{O}_{2}$ stimulation or high glucose stress on miR-195 regulation (39). By contrast, miR-195 downregulation was coupled with a diminishing effect on MDA and promoting effect on SOD; this was consistent with results reported by Liu et al (40).

Brain-derived neurotrophic factor and $\mathrm{Bcl}-2$ like protein 2 are downstream target genes of miR-195 $(32,33)$, and the present study demonstrated that RORA mediated cardioprotection of miR-195 knockdown against hypoxia-mediated oxidative injury in AC16 cells by serving as a downstream target of miR-195. The present study confirmed RORA downregulation in the serum of patients with AMI and hypoxia-treated AC16 cells, indicating a potential role of RORA in myocardial anoxia. Moreover, RORA, along with seven other genes, has been identified as a key gene in myocardial infarction, according to RNA sequencing data (41). He et al (20) identified the protective role of RORA in myocardial I/R injury, and proposed that RORA was downregulated in a mouse heart following I/R. Deficiency of RORA exacerbates high fat diet-induced myocardial hypertrophy, fibrosis and dysfunction (42). Beak et al (19) also reported angiotensin II-stimulated cardiac hypertrophy and cardiomyocyte death were exaggerated following RORA loss, accompanied by increased inflammatory response and decreased oxidative stress. Additionally, overexpression of RORA is considered to prevent oxidative damage in different 
types of cells, such as neurons (43) and hepatocytes (44), as well as cardiomyocytes $(19,20)$. The present study demonstrated that RORA ectopic expression levels attenuated ROS and MDA levels, the apoptosis rate and the expression levels of Bax and cleaved casp-3 but increased SOD activity and the expression levels of SOD2 and GPx1 in hypoxic AC16 cells.

Cathepsins (Cat) are matrix degradation proteases that are considered to modulate vascular aging and remodeling. For example, Xin et al (45) revealed that matrix metalloproteinase (MMP)-2, MMP-9, Cat S and Cat K were upregulated in response to chronic stress-induced aortic senescence, accompanied by oxidative and inflammatory responses in human umbilical vein endothelial cells. The peroxisome proliferator-activated receptor- $\gamma$ is upstream of Cat S signaling in pulmonary vascular remodeling (46). The Cat K/caspase- 8 axis is a key initial step for oxidative stress (47), and Cat K ablation is known to mitigate high-fat-diet-induced cardiomyocyte apoptosis and cardiac hypertrophy (48). However, the role of the FGD5-AS1/miR-195/RORA axis in Cat S/K expression levels was not determined in the present study, although previous studies have demonstrated that Cat $\mathrm{S}$ and Cat $\mathrm{K}$ are downregulated in intermittent hypoxia-treated and ischemia-induced mice, respectively $(49,50)$. Moreover, Cat K has been revealed to promote proliferation, migration and tube formation of human vascular endothelial cells under hypoxia via acting as target of miR-185-5p and the Notch 1 signaling pathway $(50,51)$. Further investigation is required to determine the association between hypoxia-induced oxidative injury, the FGD5-AS1/miR-195/RORA axis, cardiomyocytes and Cat $\mathrm{S} / \mathrm{K}$ expression levels.

FGD5-AS1 (co-expressing with seven other lncRNAs) is secreted in the blood of patients with AMI (11); to the best of our knowledge, however, there are no previous studies describing the source and distribution of FGD5-AS1 in vascular wall cells. miR-195 is be secreted by endothelial cells of the pulmonary and carotid arteries into smooth muscle cells $(52,53)$, indicating endothelial cells are the source of miR-195 in vascular walls. RORA is present in human vascular endothelial cells of the umbilical vein and lung microvessels $(54,55)$; moreover, higher expression levels of RORA isoform 1 have been detected in human smooth muscle cells of the aorta, compared with those of endothelial cells, mammary arteries and atherosclerotic plaques (56). However, smooth muscle cells cannot be considered as the source of RORA as high expression of RORA isoform 4 is predominantly found in endothelial cells (55). Little is known about the expression levels of FGD5-AS1, miR-195 and RORA in vascular epithelial cells. RORA and miR-195 are expressed in normal cardiomyocytes $(57,58)$, and higher levels of FGD5-AS1 and RORA (isoform 1), as well as lower miR-195 levels were observed in normoxic cardiomyocytes than in hypoxic cells. To the best of our knowledge, the present study is the first to determine FGD5-AS1 expression levels in cardiomyocytes.

In conclusion, the present study demonstrated that FGD5-AS1, which is positively correlated to RORA, suppressed oxidative stress and apoptosis in AC16 cells under hypoxia via acting as a miR-195 sponge. The results indicated a novel FGD5-AS1/miR-195/RORA axis in hypoxic injury of cardiomyocytes in vitro, which may be a potential therapeutic target against AMI by lowering oxidative damage.

\section{Acknowledgements}

Not applicable.

\section{Funding}

The present study was supported by the Jiangxi Provincal Natural Science Foundation (grant no. 20171BAB215005).

\section{Availability of data and materials}

The datasets used and/or analyzed during the current study are available from the corresponding author on reasonable request.

\section{Authors' contributions}

$\mathrm{XC}, \mathrm{SW}$ and LH conceptualized and designed the experiments, performed the data collection and analysis, and prepared the original draft of the manuscript. PZ, SY, BL and $\mathrm{HZ}$ performed data validation and checked the manuscript. XY and LS collected and analyzed the data, and revised the paper critically for important intellectual content prior to reviewing and editing. All authors read and approved the final manuscript.

\section{Ethics approval and consent to participate}

The present study was approved by the Ethics Review Committee of Jiangxi Provincial People's Hospital Affiliated to Nanchang University (Nanchang, China).

\section{Patient consent for publication}

Not applicable.

\section{Competing interests}

The authors declare that they have no competing interests.

\section{References}

1. GBD 2013 Mortality and Causes of Death Collaborators: Global, regional, and national age-sex specific all-cause and cause-specific mortality for 240 causes of death, 1990-2013: A systematic analysis for the global burden of disease study 2013. Lancet 385: 117-171, 2015.

2. Lim GB: Acute coronary syndromes: Supplemental oxygen in myocardial infarction. Nat Rev Cardiol 14: 632, 2017.

3. Ham PB III and Raju R: Mitochondrial function in hypoxic ischemic injury and influence of aging. Prog Neurobiol 157: 92-116, 2017.

4. Yu B, Meng F, Yang Y, Liu D and Shi K: NOX2 antisense attenuates hypoxia-induced oxidative stress and apoptosis in cardiomyocyte. Int J Med Sci 13: 646-652, 2016.

5. Hou L, Guo J, Xu F, Weng X, Yue W and Ge J: Cardiomyocyte dimethylarginine dimethylaminohydrolasel attenuates left-ventricular remodeling after acute myocardial infarction: Involvement in oxidative stress and apoptosis. Basic Res Cardiol 113: 28, 2018

6. Zhang Y, Li C, Meng H, Guo D, Zhang Q, Lu W, Wang Q, Wang Y and Tu P: BYD ameliorates oxidative stress-induced myocardial apoptosis in heart failure post-acute myocardial infarction via the P38 MAPK-CRYAB signaling pathway. Front Physiol 9: 505, 2018.

7. Lucas T, Bonauer A and Dimmeler S: RNA therapeutics in cardiovascular disease. Circ Res 123: 205-220, 2018

8. Kaikkonen MU, Halonen P, Liu OH, Turunen TA, Pajula J, Moreau P, Selvarajan I, Tuomainen T, Aavik E, Tavi P and Ylä-Herttuala S: Genome-wide dynamics of nascent noncoding RNA transcription in porcine heart after myocardial infarction. Circ Cardiovasc Genet 10: e001702, 2017. 
9. Li H, Cheng Z, Tang Y, Feng M, Yin A, Zhang H, Xu J, Zhang Q, Zhang J and Qian L: Expression profile of long noncoding RNAs in cardiomyocytes exposed to acute ischemic hypoxia. Mol Med Rep 19: 302-308, 2019.

10. Ong SB, Katwadi K, Kwek XY, Ismail NI, Chinda K, Ong SG and Hausenloy DJ: Non-coding RNAs as therapeutic targets for preventing myocardial ischemia-reperfusion injury. Expert Opin Ther Targets 22: 247-261, 2018.

11. Shen LS, Hu XF, Chen T, Shen GL and Cheng D: Integrated network analysis to explore the key mRNAs and lncRNAs in acute myocardial infarction. Math Biosci Eng 16: 6426-6437, 2019.

12. Liu H, Xu D, Zhong X, Xu D, Chen G, Ge J and Li H: LncRNA-mRNA competing endogenous RNA network depicts transcriptional regulation in ischaemia reperfusion injury. J Cell Mol Med 23: 2272-2276, 2019.

13. Li D, Jiang X, Zhang X, Cao G, Wang D and Chen Z: Long noncoding RNA FGD5-AS1 promotes colorectal cancer cell proliferation, migration, and invasion through upregulating CDCA7 via sponging miR-302e. In Vitro Cell Dev Biol Anim 55: 577-585, 2019.

14. Lei Y, Shi Y, Duan J, Liu Y, Lv G, Shi R, Zhang F, Yang Q and Zhao W: Identification of alternative splicing and lncRNA genes in pathogenesis of small cell lung cancer based on their RNA sequencing. Adv Clin Exp Med 28: 1043-1050, 2019.

15. Zhu H, Lu J, Zhao H, Chen Z, Cui Q, Lin Z, Wang X, Wang J, Dong H, Wang S and Tan J: Functional long noncoding RNAs (lncRNAs) in clear cell kidney carcinoma revealed by reconstruction and comprehensive analysis of the lncRNA-miRNA-mRNA regulatory network. Med Sci Monit 24: 8250-8263, 2018

16. Yu W, Liang X, Li X, Zhang Y, Sun Z, Liu Y and Wang J: MicroRNA-195: A review of its role in cancers. Onco Targets Ther 11: 7109-7123, 2018

17. Katoh M: Cardio-miRNAs and onco-miRNAs: Circulating miRNA-based diagnostics for non-cancerous and cancerous diseases. Front Cell Dev Biol 2: 61, 2014.

18. van Rooij E, Sutherland LB, Liu N, Williams AH, McAnally J, Gerard RD, Richardson JA and Olson EN: A signature pattern of stress-responsive microRNAs that can evoke cardiac hypertrophy and heart failure. Proc Natl Acad Sci USA 103: 18255-18260, 2006.

19. Beak JY, Kang HS, Huang W, Myers PH, Bowles DE, Jetten AM and Jensen BC: The nuclear receptor ROR $\alpha$ protects against angiotensin II-induced cardiac hypertrophy and heart failure. Am J Physiol Heart Circ Physiol 316: H186-H200, 2019.

20. He B, Zhao Y, Xu L, Gao L, Su Y, Lin N and Pu J: The nuclear melatonin receptor $\mathrm{ROR} \alpha$ is a novel endogenous defender against myocardial ischemia/reperfusion injury. J Pineal Res 60: 313-326, 2016

21. Livak KJ and Schmittgen TD: Analysis of relative gene expression data using real-time quantitative PCR and the 2(-Delta Delta C(T)) method. Methods 25: 402-408, 2001

22. Bar-Or D, Bar-Or R, Rael LT and Brody EN: Oxidative stress in severe acute illness. Redox Biol 4: 340-345, 2015.

23. Wei Q, Zhou HY, Shi XD, Cao HY and Qin L: Long noncoding RNA NEAT1 promotes myocardiocyte apoptosis and suppresses proliferation through regulation of miR-129-5p. J Cardiovasc Pharmacol 74: 535-541, 2019.

24. Hu H, Wu J, Yu X, Zhou J, Yu H and Ma L: Long non-coding RNA MALAT1 enhances the apoptosis of cardiomyocytes through autophagy inhibition by regulating TSC2-mTOR signaling. Biol Res 52: 58, 2019

25. Lu W, Zhu L, Ruan ZB, Wang MX, Ren Y and Li W: HOTAIR promotes inflammatory response after acute myocardium infarction by upregulating RAGE. Eur Rev Med Pharmacol Sci 22 7423-7430, 2018

26. Su Q, Liu Y, Lv XW, Dai RX, Yang XH and Kong BH: LncRNA TUG1 mediates ischemic myocardial injury by targeting miR-132-3p/HDAC3 axis. Am J Physiol Heart Circ Physiol 318: H332-H344, 2020

27. Jiao L, Li M, Shao Y, Zhang Y, Gong M, Yang X, Wang Y, Tan Z, Sun L, Xuan L, et al: lncRNA-ZFAS1 induces mitochondria-mediated apoptosis by causing cytosolic $\mathrm{Ca}^{2+}$ overload in myocardial infarction mice model. Cell Death Dis 10: 942, 2019

28. Chen H, Lan Z, Li Q and Li Y: Abnormal expression of long noncoding RNA FGD5-AS1 affects the development of periodontitis through regulating miR-142-3p/SOCS6/NF- $\mathrm{kB}$ pathway. Artif Cells Nanomed Biotechnol 47: 2098-2106, 2019.

29. Cheng WP, Lo HM, Wang BW, Chua SK, Lu MJ and Shyu KG: Atorvastatin alleviates cardiomyocyte apoptosis by suppressing TRB3 induced by acute myocardial infarction and hypoxia. J Formos Med Assoc 116: 388-397, 2017.
30. Li S, Liu X, Li H, Pan H, Acharya A, Deng Y, Yu Y, Haak R, Schmidt J, Schmalz G and Ziebolz D: Integrated analysis of long noncoding RNA-associated competing endogenous RNA network in periodontitis. J Periodontal Res 53: 495-505, 2018.

31. He JF, Luo YM, Wan XH and Jiang D: Biogenesis of MiRNA-195 and its role in biogenesis, the cell cycle, and apoptosis. J Biochem Mol Toxicol 25: 404-408, 2011

32. Hang P, Sun C, Guo J, Zhao J and Du Z: BDNF-mediates Down-regulation of MicroRNA-195 inhibits ischemic cardiac apoptosis in rats. Int J Biol Sci 12: 979-989, 2016.

33. Zhang N, Meng X, Mei L, Hu J, Zhao C and Chen W: The long non-coding RNA SNHG1 attenuates cell apoptosis by regulating miR-195 and BCL2-like protein 2 in human cardiomyocytes. Cell Physiol Biochem 50: 1029-1040, 2018

34. Gao CK, Liu H, Cui CJ, Liang ZG, Yao H and Tian Y: Roles of MicroRNA-195 in cardiomyocyte apoptosis induced by myocardial ischemia-reperfusion injury. J Genet 95: 99-108, 2016.

35. You XY, Huang JH, Liu B, Liu SJ, Zhong Y and Liu SM: HMGA1 is a new target of miR-195 involving isoprenaline-induced cardiomyocyte hypertrophy. Biochemistry (Mosc) 79: 538-544, 2014.

36. Wang L, Qin D, Shi H, Zhang Y, Li H and Han Q: MiR-195-5p promotes cardiomyocyte hypertrophy by targeting MFN2 and FBXW7. Biomed Res Int 2019: 1580982, 2019.

37. Zheng D, Ma J, Yu Y, Li M, Ni R, Wang G, Chen R, Li J, Fan GC, Lacefield JC and Peng T: Silencing of miR-195 reduces diabetic cardiomyopathy in C57BL/6 mice. Diabetologia 58: 1949-1958, 2015.

38. Zhu H, Yang Y, Wang Y, Li J, Schiller PW and Peng T: MicroRNA-195 promotes palmitate-induced apoptosis in cardiomyocytes by down-regulating Sirt1. Cardiovasc Res 92: 75-84, 2011.

39. Zhang R, Garrett Q, Zhou H, Wu X, Mao Y, Cui X, Xie B, Liu Z, Cui D, Jiang L, et al: Upregulation of miR-195 accelerates oxidative stress-induced retinal endothelial cell injury by targeting mitofusin 2 in diabetic rats. Mol Cell Endocrinol 452: 33-43, 2017.

40. Liu P, Peng QH, Tong P and Li WJ: Astragalus polysaccharides suppresses high glucose-induced metabolic memory in retinal pigment epithelial cells through inhibiting mitochondrial dysfunction-induced apoptosis by regulating miR-195. Mol Med 25: 21, 2019.

41. Zhao Q, Wu K, Li N, Li Z and Jin F: Identification of potentially relevant genes for myocardial infarction using RNA sequencing data analysis. Exp Ther Med 15: 1456-1464, 2018.

42. Zhao YC, Xu LW, Ding S, Ji QQ, Lin N, He Q, Gao LC, Su YY, $\mathrm{Pu} \mathrm{J}$ and $\mathrm{He} \mathrm{B}$ : Nuclear receptor retinoid-related orphan receptor alpha deficiency exacerbates high-fat diet-induced cardiac dysfunction despite improving metabolic abnormality. Biochim Biophys Acta Mol Basis Dis 1863: 1991-2000, 2017.

43. Boukhtouche F, Vodjdani G, Jarvis CI, Bakouche J, Staels B, Mallet J, Mariani J, Lemaigre-Dubreuil Y and Brugg B: Human retinoic acid receptor-related orphan receptor alphal overexpression protects neurones against oxidative stress-induced apoptosis. J Neurochem 96: 1778-1789, 2006.

44. Han YH, Kim HJ, Kim EJ, Kim KS, Hong S, Park HG and Lee MO: ROR $\alpha$ decreases oxidative stress through the induction of SOD2 and GPx1 expression and thereby protects against nonalcoholic steatohepatitis in mice. Antioxid Redox Signal 21: 2083-2094, 2014

45. Xin M, Jin X, Cui X, Jin C, Piao L, Wan Y, Xu S, Zhang S, Yue X, Wang H, et al: Dipeptidyl peptidase-4 inhibition prevents vascular aging in mice under chronic stress: Modulation of oxidative stress and inflammation. Chem Biol Interact 314: 108842, 2019

46. Chang CJ, Hsu HC, Ho WJ, Chang GJ, Pang JS, Chen WJ, Huang CC and Lai YJ: Cathepsin S promotes the development of pulmonary arterial hypertension. Am J Physiol Lung Cell Mol Physiol 317: L1-L13, 2019.

47. Hu L, Huang Z, Ishii H, Wu H, Suzuki S, Inoue A, Kim W, Jiang H, Li X, Zhu E, et al: PLF-1 (Proliferin-1) modulates smooth muscle cell proliferation and development of experimental intimal hyperplasia. J Am Heart Assoc 8: e005886, 2019.

48. Hua Y, Zhang Y, Dolence J, Shi GP, Ren J and Nair S: Cathepsin $\mathrm{K}$ knockout mitigates high-fat diet-induced cardiac hypertrophy and contractile dysfunction. Diabetes 62: 498-509, 2013.

49. Yang YY, Li LY, Jiao XL, Jia LX, Zhang XP, Wang YL, Yang S, Li J, Du J, Wei YX and Qin YW: Intermittent hypoxia alleviates $\beta$-aminopropionitrile monofumarate induced thoracic aortic dissection in C57BL/6 mice. Eur J Vasc Endovasc Surg 59: $1000-1010,2020$ 
50. Jiang H, Cheng XW, Shi GP, Hu L, Inoue A, Yamamura Y, Wu H, Takeshita K, Li X, Huang Z, et al: Cathepsin K-mediated Notch1 activation contributes to neovascularization in response to hypoxia. Nat Commun 5: 3838, 2014.

51. Li CC, Qiu XT, Sun Q, Zhou JP, Yang HJ, Wu WZ, He LF, Tang CE, Zhang GG and Bai YP: Endogenous reduction of miR-185 accelerates cardiac function recovery in mice following myocardial infarction via targeting of cathepsin K. J Cell Mol Med 23: 1164-1173, 2019.

52. Zeng Z, Yao J,Li Y, Xue Y,Zou Y, Shu Zand Jiao Z: Anti-apoptosis endothelial cell-secreted microRNA-195-5p promotes pulmonary arterial smooth muscle cell proliferation and migration in pulmonary arterial hypertension. J Cell Biochem 119: 2144-2155, 2018.

53. Gu J, Zhang H, Ji B, Jiang H, Zhao T, Jiang R, Zhang Z, Tan S Ahmed A and Gu Y: Vesicle miR-195 derived from endothelial cells inhibits expression of serotonin transporter in vessel smooth muscle cells. Sci Rep 7: 43546, 2017.

54. Gulec C, Coban N, Ozsait-Selcuk B, Sirma-Ekmekci S, Yildirim $\mathrm{O}$ and Erginel-Unaltuna N: Identification of potential target genes of ROR-alpha in THP1 and HUVEC cell lines. Exp Cell Res 353: 6-15, 2017.
55. Kim SY, Kim HJ, Park MK, Huh JW, Park HY, Ha SY, Shin JH and Lee YS: Mitochondrial E3 ubiquitin protein ligase 1 mediates cigarette smoke-induced endothelial cell death and dysfunction. Am J Respir Cell Mol Biol 54: 284-296, 2016.

56. Besnard S, Heymes C, Merval R, Rodriguez M, Galizzi JP, Boutin JA, Mariani J and Tedgui A: Expression and regulation of the nuclear receptor RORalpha in human vascular cells. FEBS Lett 511: 36-40, 2002.

57. Xu L, Su Y, Zhao Y, Sheng X, Tong R, Ying X, Gao L, Ji Q, Gao Y, Yan Y, et al: Melatonin differentially regulates pathological and physiological cardiac hypertrophy: Crucial role of circadian nuclear receptor ROR $\alpha$ signaling. J Pineal Res 67: e12579, 2019.

58. Porrello ER, Johnson BA, Aurora AB, Simpson E, Nam YJ, Matkovich SJ, Dorn GW II, van Rooij E and Olson EN: MiR-15 family regulates postnatal mitotic arrest of cardiomyocytes. Circ Res 109: 670-679, 2011.

This work is licensed under a Creative Commons Attribution-NonCommercial-NoDerivatives 4.0 International (CC BY-NC-ND 4.0) License. 